\section{What is causing the corneal ulcer? Management strategies for unresponsive corneal ulceration}

\begin{abstract}
Infectious keratitis represents a significant cause of ocular morbidity in the United States. The work-up and treatment of presumed infectious keratitis (PIK) has changed in the past two decades. The development of newer topical antibiotics has enabled broad-spectrum antibiotic coverage with good tissue penetration. The majority of PIK cases respond well to this strategy. The small numbers of cases that do not respond to the treatment are the cases that offer a diagnostic and therapeutic challenge. This review will describe different algorithms that can be followed for the successful management of patients with difficult or progressive PIK. These algorithms are based on scientific work and on our empirical clinical experience. The review will also present three different clinical cases of PIK that were managed according to the algorithms presented in this review.
\end{abstract}

Bascom Palmer Eye Institute, University of Miami Miller School of Medicine, Miami, FL, USA

Correspondence: G Amescua, Bascom Palmer Eye Institute, University of Miami, Miller School of Medicine, Miami 33136, $\mathrm{FL}$, USA

Tel: + 1305326 6000;

Fax: + 13054824853

E-mail: gamescua@med. miami.edu

Received: 13 September 2011

Accepted in revised form: 28 October 2011

Published online: 9

December 2011
Eye (2012) 26, 228-236; doi:10.1038/eye.2011.316;

published online 9 December 2011

Keywords: corneal ulcer; infectious keratatis treatment; approach to corneal ulcers

\section{Introduction}

Infectious keratitis represents a significant cause of ocular morbidity in the United States with historic incidence of about 30000 cases per year. ${ }^{1}$ In California, an incidence of 27.6 per 100000 person/year was reported, for an estimate of 75000 corneal ulcers annually in the United States. ${ }^{2}$ The conservative estimate of the number of corneal ulcers occurring annually in the developing world is around 1.5 million cases a year. ${ }^{3}$ The incidence of bacterial keratitis
G Amescua, D Miller and EC Alfonso

in the United States accounts for 3 of every 1000 patients seen by an ophthalmologist. ${ }^{4}$ The risk factors for corneal ulceration are trauma, contact lens wear, recent corneal surgery, ocular surface disease, chronic use of topical medication, and systemic immunosuppresion..$^{5}$ About $50 \%$ of the patients who develop an infectious keratitis will obtain an optimal vision of 20/60 or worse, and $25 \%$ of those patients will attain a final vision of $20 / 200{ }^{4}$

The work up and treatment in the United States for patients with presumed infectious keratitis (PIK) has changed in the past two decades. With the development of newer topical antibiotics regimens, especially with the development of the third- and fourthgeneration fluoroquinolones it is possible to offer empiric broad-spectrum antibiotic coverage with good tissue penetration. ${ }^{6,7}$ Even though the management of PIK varies between a general ophthalmology and a cornea subspecialty practice, the first line of treatment for PIK continues to be a fourth-generation fluoroquinolone in the United States. Only 12\% of the general ophthalmology practitioners use fortified antibiotics as the initial choice of treatment compared with $41 \%$ of the corneal subspecialists that use a combination of fortified antibiotics and a fouth-generation fluoroquinolone. In the United States, most cases of PIK are treated with an empirical approach using monotherapy with a fourthgeneration fluoroquinolone. Microbiology studies are rarely used when patients present to comprehensive ophthalmology practices. The majority of the PIK cases respond well to this type of management. ${ }^{8}$ The small numbers of cases that do not respond to the treatment are the cases that offer a diagnostic and therapeutic challenge to the corneal specialist. 
In general, the management algorithms for PIK can be divided into three: (1) a culture-guided approach, with cultures obtained in every patient; (2) an empirical approach, where patients are started on a broadspectrum antibiotic, and if the patient does not respond, then the need for diagnostic and microbiology studies or change in the antibiotic coverage is evaluated; (3) a case-based approach, where the treatment is decided based on the location and severity of the corneal ulcers. ${ }^{9}$ The main goal if this review is to describe the algorithms that are followed at our academic institution for the management of patients with difficult or progressive PIK. These algorithms consider our regional epidemiological and microbiological data and the regional availability of ophthalmic antimicrobials.

At our institution, we care for of a large number of patients with PIK. In 2010, a total of 736 patients presented with a clinical diagnosis of PIK that prompted the clinician toward obtaining microbiology workup. Of the 736 patients, 280 (38\%) of the cases had a positive organism isolated. About $55 \%$ of the patients were already on treatment with topical antimicrobials.

When a patient presents for evaluation of a PIK, the clinical history and clinical findings categorizes the patient as a low risk $v s$ a high risk. High-risk characteristics are a history of contact lens use, history of trauma, history of recurrent topical steroid use, history of immunosuppression, and history of ocular surface disease. Patients who are at low risk have no history of contact lens use, no history of trauma, and no history of recurrent topical medication use. In the following section we present three different algorithms for different clinical scenarios of patients presenting with PIK. If there is significant thinning or perforation, microbiology studies are obtained and corneal gluing is attempted. If this is unsuccessful, surgical repair with keratoplasty is indicated. Either therapeutic full-thickness, lamellar graft, or corneal patch graft can be attempted. ${ }^{10}$ It is important that the tissue obtained during surgery is sent for identification of microorganism by microbiological culture and histopathology. ${ }^{11}$ At our institution, the microbiology and pathology records from 425 consecutive corneal specimens, including 42 corneal biopsies and 383 keratoplasty specimens, were evaluated for the presence of bacteria, fungi, acanthamoeba, and mycobacteria detected either by histological staining or by growth in culture. In $45 \%$ of the cases an organism was isolated. Fungi were present in $43 \%$ of the cases, bacteria in $39 \%$, and acanthamoeba in $14 \%$ and mycobacteria in $4 \% .{ }^{12}$ Also in every patient evaluated with PIK that presents with a keratitis extending to the sclera, corneal limbal area and scleral tissue, cultures should be obtained and sent to microbiology. An association with a systemic disease should be considered.

\section{Algorithm no. 1}

Low-risk patients (Figure 1) are cultured ${ }^{13}$ (blood agar, chocolate agar, thioglycolate broth, and Saborauds agar), and confocal microscopy may be indicated when the clinician is suspicious for a fungal or a parasitic infection. ${ }^{14}$ For smears, we recommend obtaining a Gram and a Giemsa stain. Agar-agar plate is sent only when there is suspicion for parasitic infection. If there are risk factors for a mycobacterial infection, an acid-fast stain and Lowenstein-Jensen media is used. ${ }^{15}$ Fortified antibiotics for Gram-positive coverage and a fourthgeneration fluoroquinolone for a broad-spectrum Gram-negative coverage is recommended. We recommend applying the topical antimicrobial every hour during the first $48 \mathrm{~h}$ of treatment. If after $48-72 \mathrm{~h}$ from antimicrobial initiation the patient has clinical improvement and a positive bacterial organism is isolated and identified in smears, we strongly consider adding topical steroids with close follow-up. The use of topical corticosteroids in conjunction with topical antimicrobials has been evaluated in a prospective multicenter trial and no safety concerns were reported. ${ }^{16}$ We believe that decreasing the acute inflammatory response with the use of topical corticosteroids increases patient comfort and this may help with treatment compliance. If no organism is identified, it is recommended to continue the antibiotics, but decreasing the frequency in order to avoid toxicity. If the clinician suspects a viral etiology, culture and/or PCR for the virus are indicated with topical and systemic antiviral treatment initiated.

When a microorganism is identified, but no clinical improvement has been seen after the appropriate antimicrobial treatment and length of treatment has been implemented, several factors need to be considered. Patient compliance with treatment should be questioned. This is an indication for admitting the patient to a health care facility. The possibility of a resistant microorganism needs to be addressed. The antibiogram needs to be reviewed. If the microorganism is sensitive to treatment but there is no improvement, the ulcer can be re-scraped for smears and culture. Confocal microscopy is indicated. When confocal microscopy is able to identify a fungal or parasitic organism, organism-guided treatment should be started. The length of treatment of these organisms is long. If the confocal results and scrapings are negative and there is no clinical improvement, PCR studies ${ }^{17}$ and corneal biopsy are indicated. If an organism is identified, treat according to the organism. If there is no organism identified and the patient with no clinical improvement, a keratoplasty is indicated submitting the tissue to pathology and microbiology. In recent years, the use of corneal collagen cross-linking (CCCL) has moved from the refractive surgery field to the corneal microbiology 


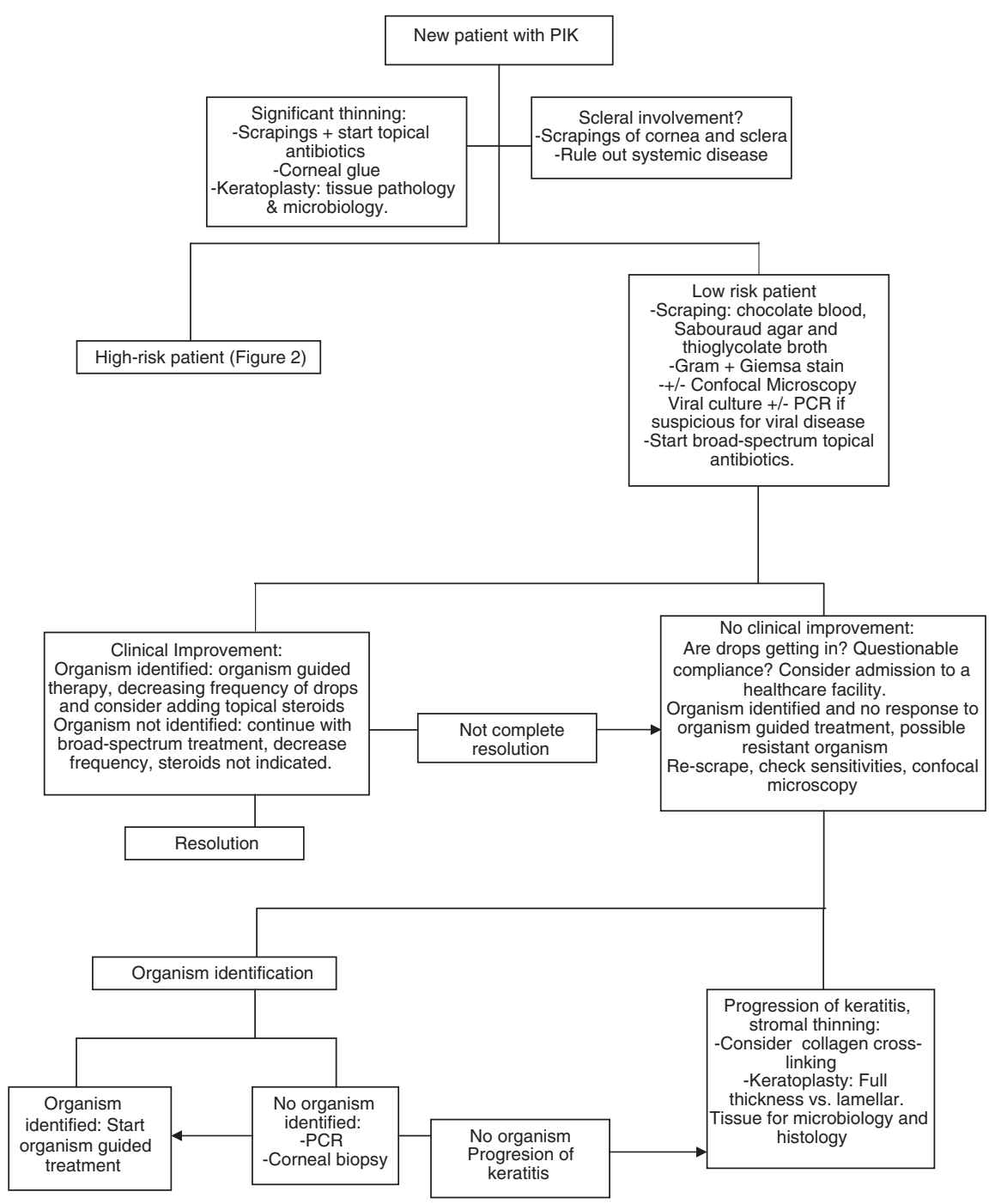

Figure 1 Bascom Palmer Eye Institute local algorithm for the diagnosis and management of the new patient with PIK and low-risk factors.

field. The photosensitization of riboflavin using ultraviolet light has the potential to induce healing in patients with infectious keratitis. ${ }^{18-20}$ This procedure may provide in the future a good therapeutic option for patients with infectious keratitis with poor response to topical antimicrobial treatment. There is a need for a prospective, randomized, and controlled trial to establish the efficacy and safety of this treatment. CCCL is not approved by the US Food and Drug Administration and not yet available at our institution.

\section{Algorithm no. 2}

The patient with (Figure 2) PIK with high-risk characteristics is also evaluated for any signs of melt or sclera extension. In these cases, scrapings for smears and cultures should be obtained for bacteria, fungi, parasites, and also mycobacteria. Gram and a Giemsa stains are sent; Calcofluor-white stain is done if there is suspicion for acanthamoeba keratitis. Culture of the contact lenses, contact lens case, contact lens solution, and confocal microscopy should be performed. Viral cultures and/or PCR should be obtained if there is a suspicion for a viral etiology. The treatment with broad spectrum antibiotics with a combination of a fortified antibiotic for Gram-positive coverage, and a fourth-generation fluoroquinolone, or a fortified aminoglycoside or a fortified third or fourth-generation cephalosporin for a broad-spectrum Gram-negative coverage is recommended. If, after the treatment has started, there is clinical improvement in a PIK case and the organism is identified, the drops should be decreased in frequency in 


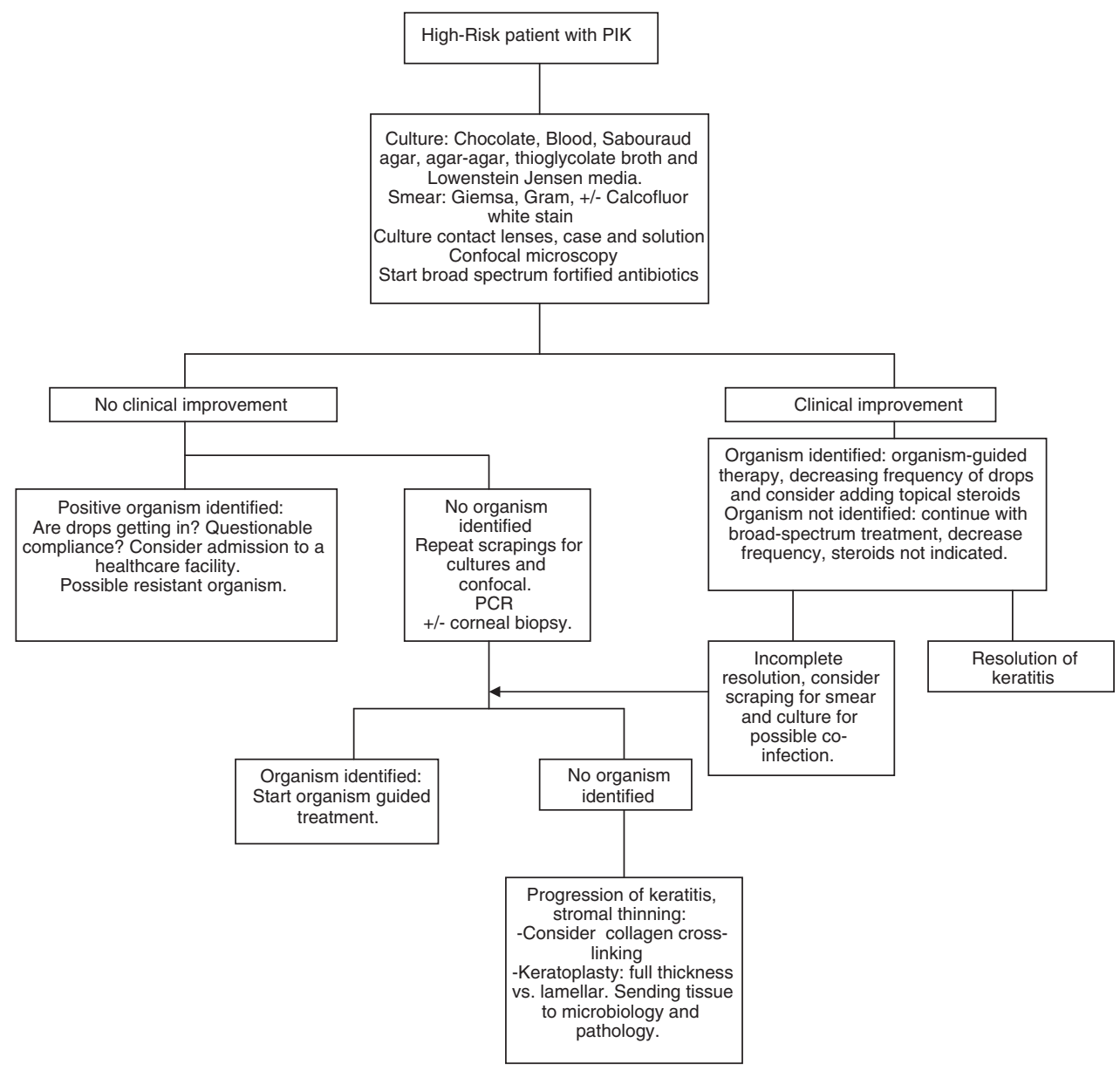

Figure 2 Bascom Palmer Eye Institute local algorithm for the diagnosis and management of a new patient with PIK and high-risk factors.

order to avoid toxicity. In cases where there is improvement but no organism is isolated, the frequency of the drops should be decreased in order to avoid toxicity. Topical steroids may be indicated to help decrease the inflammatory response. It is important not to start these until there is clinical or laboratory confirmation that the microorganisms have been eradicated. Re-scraping of the ulcer may be necessary or confocal microscopy should be performed.

If after the patient has been on topical antibiotic treatment and microbiology workup and/or confocal microscopy identified an organism but there is no clinical improvement, the possibility of a resistant organism or noncompliance with treatment should be addressed. If there is no improvement and there is no organism identified, confocal-microscopy, smears, and cultures should be repeated. PCR analysis and/or the use of a corneal biopsy should be considered. If this leads to organism identification, an organism-guided treatment should be started. If there is no organism identified and there is no significant thinning of the cornea, CCCL should be considered. If after CCCL the keratitis continues to worsen a therapeutic keratoplasty is indicated with examination of the tissue with microbiology and pathology.

\section{Algorithm no. 3}

Another scenario (Figure 3) that is very common at our institution is that of the patient who is referred to the cornea service for evaluation after being treated at the community. These patients usually have been on a broadspectrum topical antibiotic regimen and microbiological studies were rarely performed. Even though these patients have been on antibiotics, cultures are indicated. We have previously reported that positive organism identification can be obtained in patients already on topical antibiotics. ${ }^{21}$ This study showed that patients on treatment whose samples were cultured had a significant delay in starting microbiology-guided therapy and took a significantly longer time to heal, but it was possible to isolate an organism. When culturing the samples of 


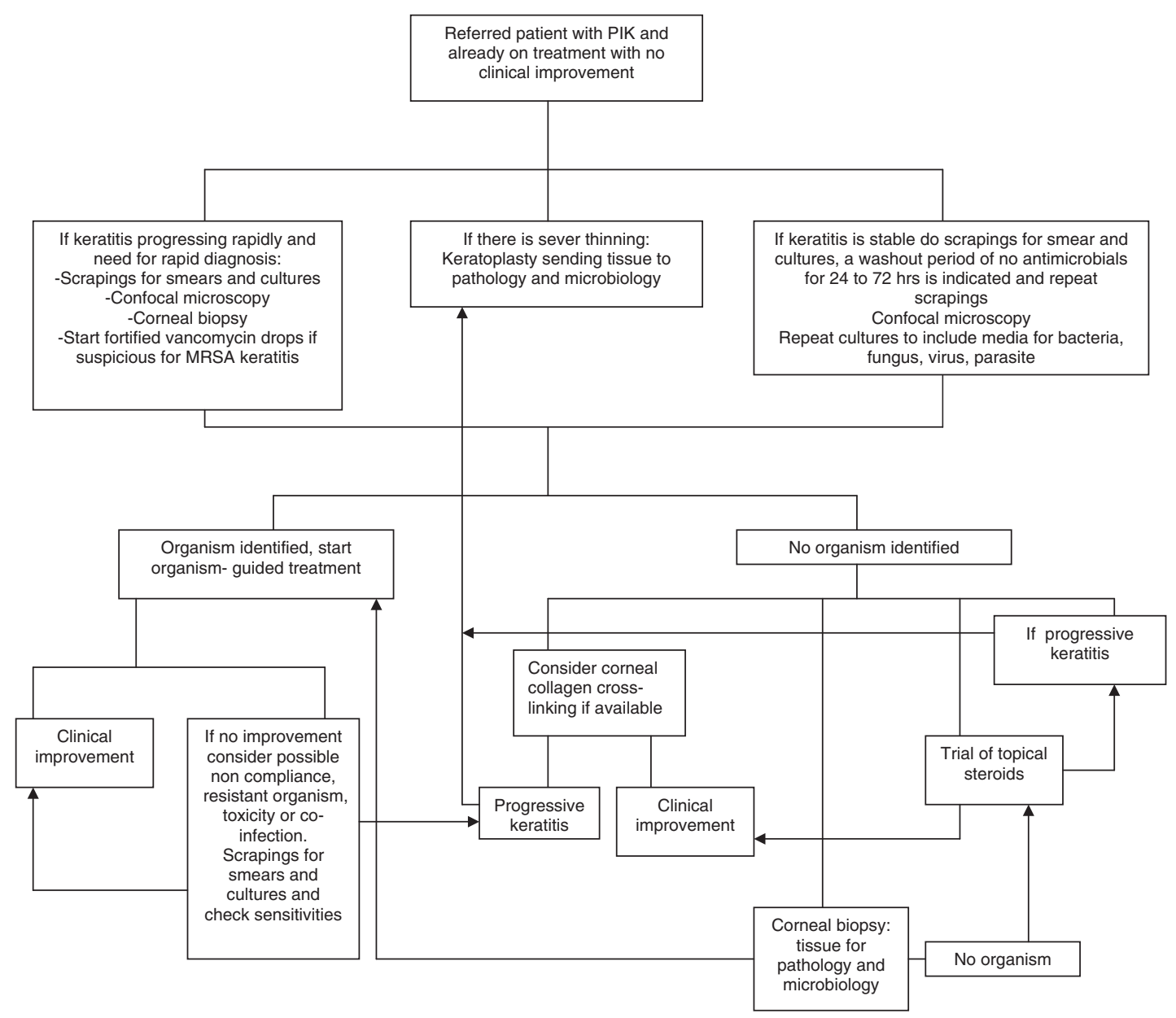

Figure 3 Bascom Palmer Eye Institute local algorithm for a patient referred for evaluation for PIK who is already on treatment and with a poor clinical response.

patients who are already on topical antibiotic treatment, the use of smears and thioglycolate broth is recommended. From June 2006 to January 2007, our microbiology laboratory reported a total of 36 organisms that were isolated only on thioglycolate broth. ${ }^{22}$ If the keratitis is stable, the recommendation is to perform confocal microscopy, and scrapings for smears and cultures, after a washout period of about $24-72 \mathrm{~h}$. PCR analysis is recommended if available. Corneal biopsy is indicated if the previous studies fail to identify an organism.

If the patient has risk factors for methicillin-resistant Staphylococcus aureus infection, the recommendation is to start fortified vancomycin drops. If there is no improvement even with broad-spectrum coverage for resistant bacteria, we recommend re-culturing and sending tissue for PCR analysis. Confocal microscopy should be repeated. If unable to isolate an organism, and no significant thinning of the cornea, CCCL can be considered. Another possibility in the management of patients who come for evaluation with history of use of multiple antimicrobials is to perform confocal microscopy and start a washout period of 24 to $72 \mathrm{~h}$ and then perform corneal scrapings for cultures. The other alternative is to start a trial of topical corticosteroids with close follow-up. If the condition of patients on a challenge trial of topical steroids worsens, cultures should be repeated and corneal biopsy or keratoplasty may be indicated.

\section{Case no. 1}

An 83-year-old woman with a history of complicated cataract surgery in her left eye presented for evaluation of painful pseudophakic corneal edema with a BCVA of hand motions. Descemet's stripping endothelial keratoplasty was performed. The patient required a repositioning of the donor tissue 10 days after the surgery. The patient's corneal edema and pain were resolved. The patient was instructed to continue on 

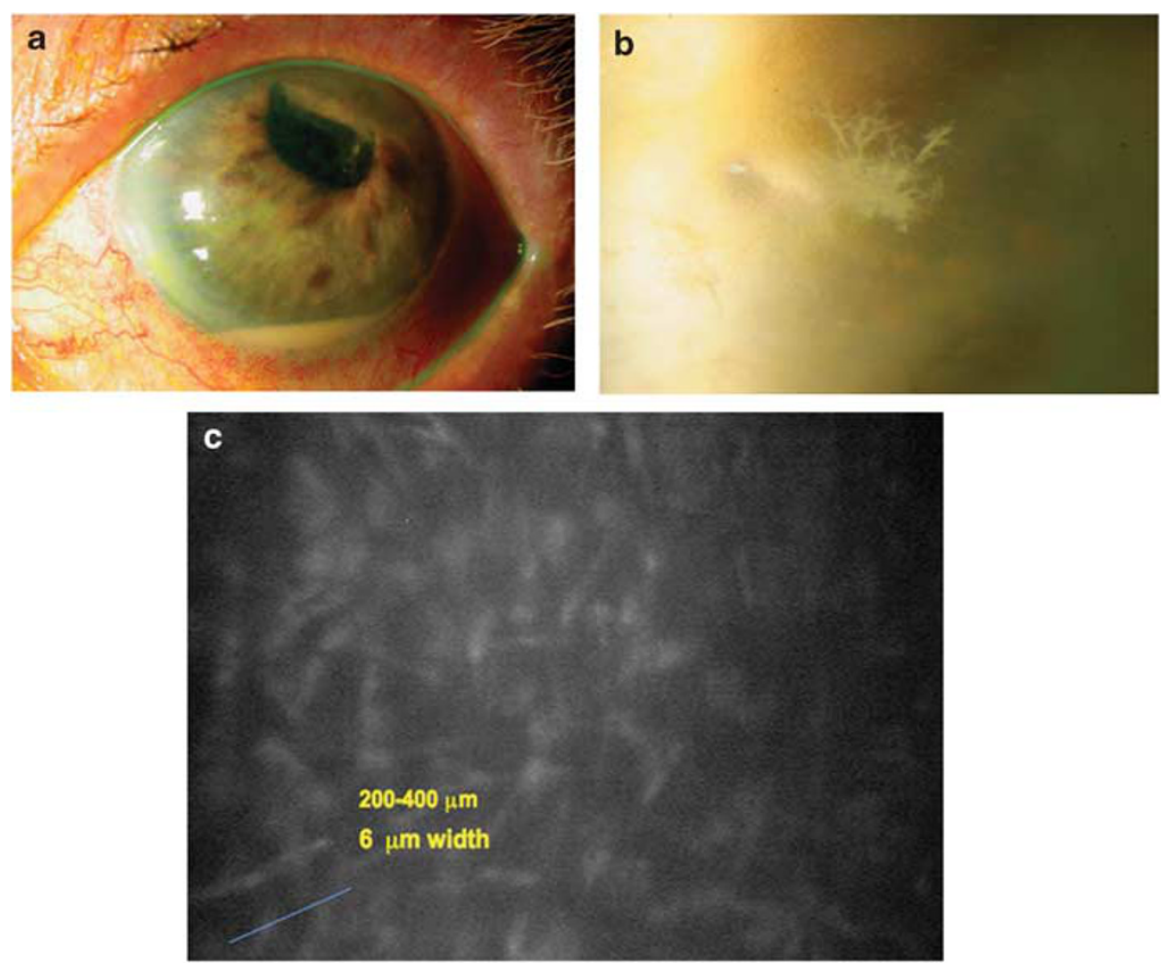

Figure 4 Photos (a) and (b) are slit lamp photos of the patient presented as Case no. 1. Photo (b) shows a high-magnification view of the 'crystalline-like infiltrate' seen on this patient after a DSEK procedure. Photo (c) shows confocal microscopy results compatible with fungal keratitis.

prednisolone acetate $1 \%$ drops four times a day. Five months later, she presented with a painful red left eye. The patient had a clear cornea with a small inferior paracentral crystalline-like infiltrate with an associated epithelial defect and a layering hypoyon (Figures $4 a$ and $b$ ). The infiltrate was located deep in the patient's left corneal stroma. No infiltrate was seen in the interface. Owing to the history of topical steroid use and the atypical infiltrate, cultures were obtained for bacteria, fungi, and mycobacteria. The patient was started on fortified vancomycin drops and moxifloxacin drops every hour. After $72 \mathrm{~h}$, patient had no clinical improvement and no organism was isolated on microbiology. Cultures were repeated and failed to identify an organism. Confocal microscopy was performed (Nidek ConfoScan 4, Nidek Co., Ltd, Gamagori, Japan). Confocal microscopy showed features compatible with fungal elements (Figure 4c). The patient was started on topical natamycin 5\% every hour while awake for the first $72 \mathrm{~h}$ and then every $2-3 \mathrm{~h}$, and 15 days later a complete resolution of the keratitis was obtained.

\section{Case no. 2}

A 16-year-old boy presented with a history of left-eye PIK treated for about 60 days with multiple antibiotics (Moxifloxacin, fortified vancomycin, and tobramycin).
The patient had a history of swimming and sleeping with his contact lenses and a history of organic trauma while camping. The patient was evaluated by multiple community ophthalmologists and received topical treatment for presumed bacterial keratitis and topical (Trifluridine) and systemic (acyclovir) treatment for presumed viral keratitis. On presentation to our institution, the patient's left-eye BCVA was 20/40. The left cornea showed a temporal paracentral superficial stromal white infiltrate with an associated epithelial defect. The patient keratitis was stable with no signs of melting or significant thinning (Figures $5 \mathrm{a}$ and b). A 72-h washout period was performed with no changes in his clinical examination. Culture and stains were obtained and no organism was identified. A femtosecond laser-assisted corneal biopsy was performed following protocol already described by our group. ${ }^{23}$ Half of the tissue was sent to the microbiology laboratory and the other half for histopathological evaluation. No organism was isolated (Figure 5c). All medications were discontinued and the patient was instructed to use preservative-free artificial tears. No topical corticosteroids were used because of the history that strongly suggested an atypical organism. The patient's infiltrate slowly improved and resolved. The last evaluation of the patient was 7 months after presentation and he showed a fully resolved keratitis. 

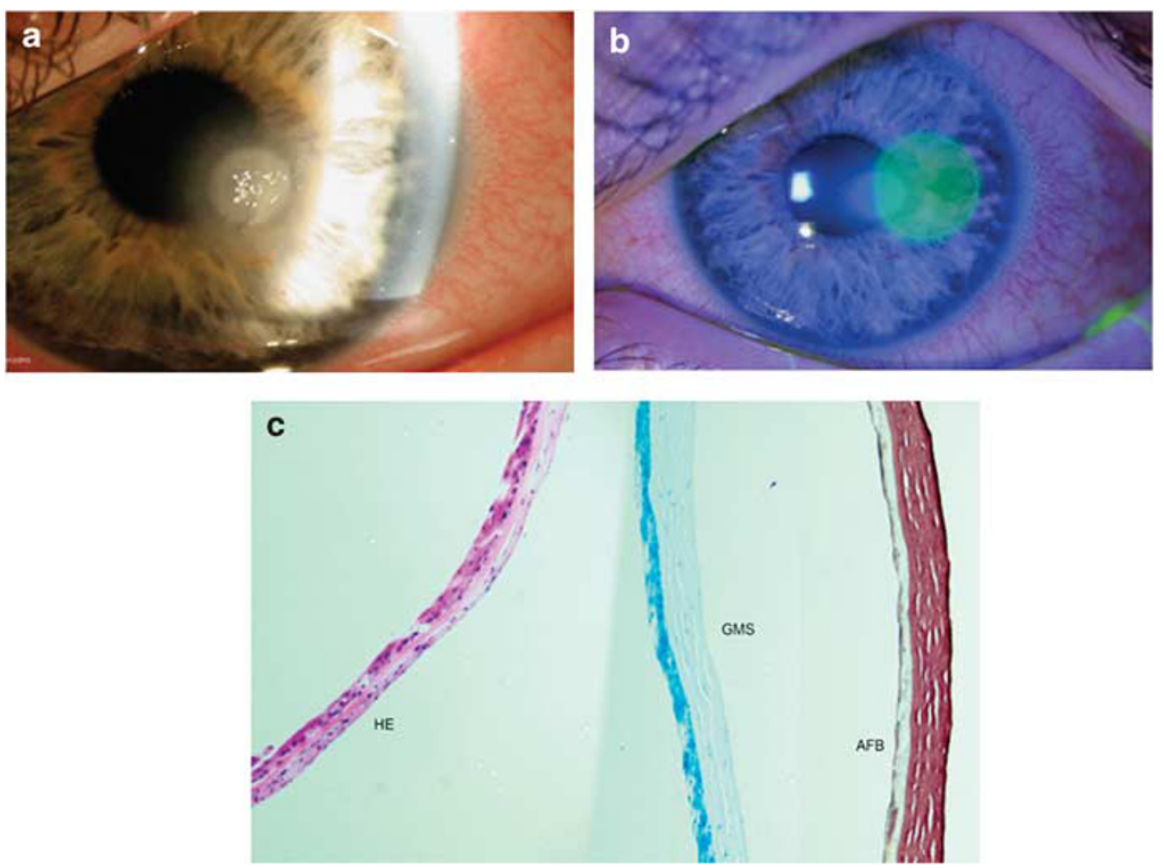

Figure 5 Photos (a) and (b) are slit lamp photos of the patient presented as Case no. 2. Photo (a) demonstrated a clinical photo of his first visit to our institution. Photo (b) shows the patient immediately after femtosecond-assisted corneal biopsy. Photo (c) shows the histology negative for microorganism.

\section{Case no. 3}

A 66-year-old man with a history of well-controlled diabetes presented to our institution for evaluation of progressive left-eye PIK. The patient had a history of swimming with his contact lenses in the coastal waters of Costa Rica. Two weeks after this trip, the patient presented to a community ophthalmologist with a left red eye. The patient was started on topical gatifloxacin drops for 2 weeks. The patient had no improvement of his signs and symptoms and topical (trifluridine) and systemic (acyclovir) antiviral treatment for presumed viral keratitis was prescribed. After a total of 1 month of treatment patient was referred to us. The patient presented with a left-eye BCVA of 20/200. The exam showed a diffuse stromal ring infiltrate (Figure 6a). His exam and history were strongly suspicious for acanthamoeba keratitis. Cultures and stains were obtained and confocal microscopy was performed. Owing to the patient's poor cooperation with the examination, the results of the confocal microscopy were inconclusive. A 3-mm corneal biopsy was performed. Tissue was sent to microbiology and pathology, $48 \mathrm{~h}$ later acanthamoeba keratitis was confirmed by the microbiology lab and histology (Figure 6b). The patient was started on double therapy with topical chlorexidine drops and topical polyhexamethylene biguanide drops. The patient was lost to $\mathrm{f} / \mathrm{u}$ and 4 weeks later presented with progressive keratitis and significant stromal thinning (Figure 6c). Stains and cultures were obtained. Paecilomyces lilacinus keratitis was identified. Owing to extension of the keratitis and the significant thinning patient was taken to the operating room for a therapeutic penetrating keratoplasty (Figure $6 \mathrm{~d}$ ). Tissue sent to pathology and microbiology confirmed the diagnosis of $P$. lilacinus (Figure 6e). The patient received an intracameral injection of voriconazol $50 \mathrm{mcg} / \mathrm{ml}$ at the end of the procedure and placed con aggressive topical voriconazol $1 \%$ drops, ${ }^{24}$ topical cyclosporine (CSA) $0.5 \%$ drops, ${ }^{25}$ atropine sulfate $1 \%$ drops, and moxifloxacin ophthalmic drops. After 2 months of treatment with topical voriconazol patient infection was controlled and patient was started on topical prednisolone acetate $1 \%$ drops four times a day and continue on CSA drops. The patient's graft failed and 6 months later a penetrating keratoplasty and an extracapsular cataract extraction was successfully performed. The patient graft has remained clear under a regimen of topical steroids and topical CSA (Figure 6f).

The diagnosis and treatment of difficult cases of microbial keratitis continues to depend on the information derived from studies that help to identify whether it's infectious or sterile, and if the former, what is the offending microorganism. This identification depends in order of priority: microbiological smears and cultures, histology, confocal microscopy, and clinical 

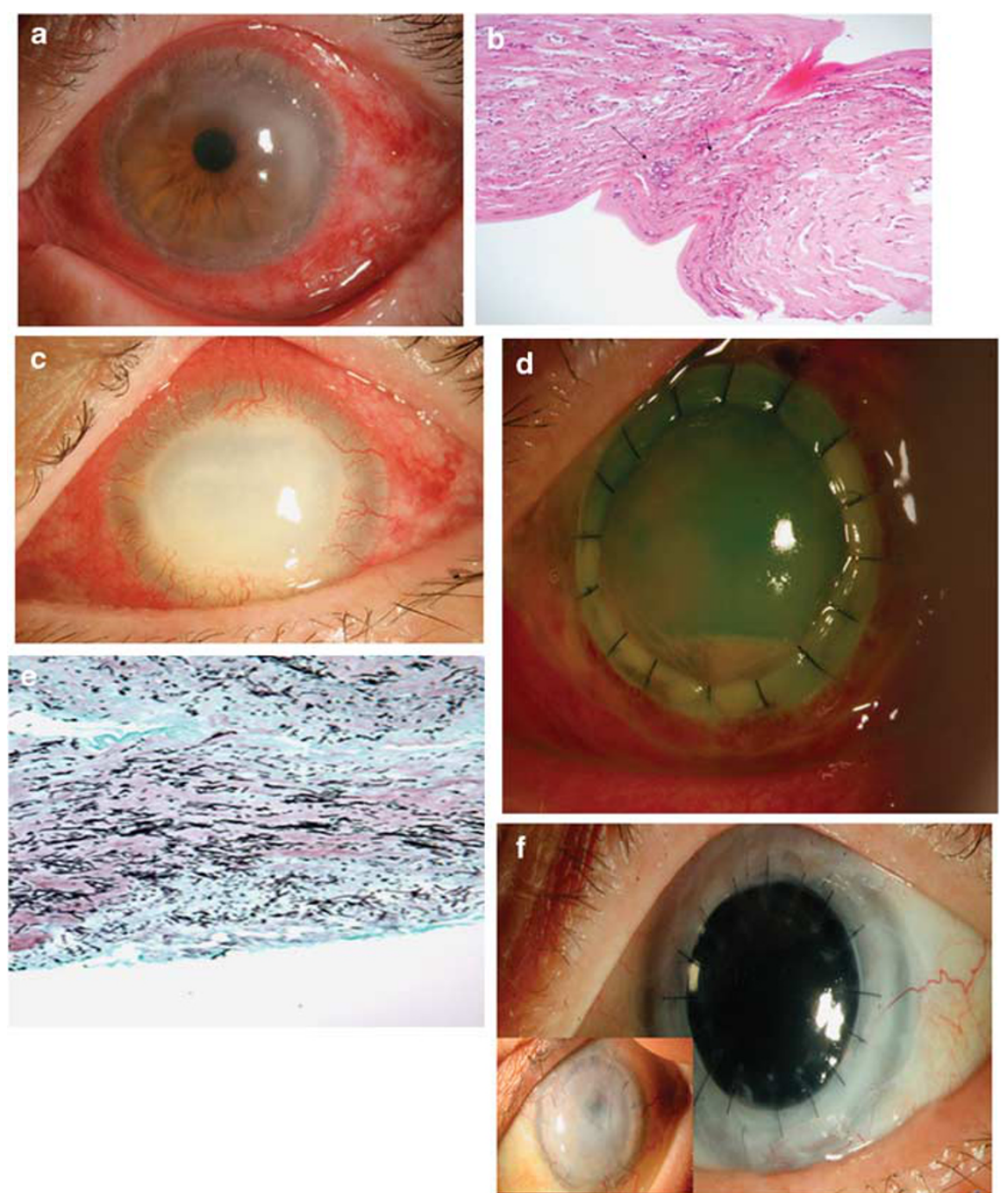

Figure 6 Photos represent the patient presented as Case no. 3. Photo (a) shows the clinical presentation as a 'ring infiltrate' type of keratitis. This photo demonstrated how the patient presented to our institution. Photo (b) demonstrated the results of the biopsy that are compatible with Acanthamoeba keratitis. The patient lost to follow-up and a month later presented with significant corneal melt and worsening keratitis (Photo c). The patient was taken to the operating room for a therapeutic corneal transplant and photo (d) shows the patient's cornea one day after graft was performed. Photo (e) demonstrated the histology results done for the cornea after the graft compatible with fungal keratitis. Photo (f) shows the patients cornea after graft at later time points. Anterior photo demonstrates graft failure 2 months after the primary intervention and the posterior photo shows a clear cornea 8 months after optical penetrating keratoplasty.

signs and symptoms. Future refinements in molecular diagnosis such as multiplex PCR and imaging may further enhance our ability to determine the cause and provide appropriate treatment. This treatment may not only include traditional antibiotic therapies, but also modulators of the inflammatory response by the microorganisms and host cornea.

A significant group of ophthalmologist and primary eye care providers in the United States do not routinely culture all their cases of infectious keratitis. ${ }^{8,26}$ More importantly, the access to the diagnostic tools described in this review are not available in multiple regions of the
World. In order to decrease the need of diagnostic test and to simplify the diagnosis of patients with PIK the use of artificial neural networks (ANN) may have a role in the future. As described by Saini et al, ${ }^{27}$ the ANN are associative self-learning techniques with the ability to identify multidimensional relationships and perform pattern recognition in nonlineal domains. Saini et al demonstrated that with the use of ANN they were able to correctly identify the category (fungal $v$ s bacterial) in 39 of 44 cases of infectious keratitis. Although initially suggested for microbial keratitis, ANN have been successfully adapted to other ocular diseases. ${ }^{28-30}$ 
In conclusion, the diagnosis and treatment of microbial keratitis continues to be a significant challenge to the ophthalmologist. We continue to look for ways that can enhance the diagnosis so that appropriate treatment can be provided, and avoid lifelong-lasting visual defects.

\section{Conflict of interest}

The authors declare no conflict of interest.

\section{References}

1 Pepose JS, Wilhelmus KR. Divergent approaches to the management of corneal ulcers. Am J Ophthalmol 1992; 114(5): 630-632.

2 Jeng BH, Gritz DC, Kumar AB. Epidemiology of ulcerative keratitis in Northern California. Arch Ophthalmol 2010; 128(8): 1022-1028.

3 Basak SK, Basak S, Mohanta A, Bhowmick A. Epidemiological and microbiological diagnosis of suppurative keratitis in Gangetic West Bengal, eastern India Indian. J Ophthalmol 2005; 53(1): 17-22.

4 Wilhelmus KR. Indecision about corticosteroids for bacterial keratitis: an evidence-based update. Ophthalmology 2002; 109(5): 835-842.

5 Cheng KH, Leung SL, Hoekman HW, Beekhuis WH, Mulder PG, Geerards AJ et al. Incidence of contact lens-associated microbial keratitis and its related morbidity. Lancet 1999; 354: 181-185.

6 O'Brien TP. Evidence based review of moxifloxacin. Int Ophthalmol Clin 2006 Fall 46(4): 61-72.

7 Chawla B, Agarwal P, Tandon R, Titiyal JS, Sharma N, Agarwal T et al. In vitro susceptibility of bacterial keratitis isolates to fourth-generation fluoroquinolones. Eur J Ophthalmol 2010; 20(2): 300-305.

8 McDonnel PJ, Nobe J, Gauderman WJ, Lee P, Aiello A, Trousdale M. Community care of corneal ulcers. Am J Ophthalmol 1992; 114: 531-538.

9 Krachmer JH, Mannis MJ, Holland EJ. Cornea: Fundamentals, Diagnosis and Treatment, vol. 1, 3rd edn. Mosby-Elsevier: Philadelphia, PA, USA; pp 936-938.

10 Sharma N, Sachdev R, Jhanji V, Titiyal JS, Vajpayee RB. Therapeutic keratoplasty for microbial keratatis. Curr Opin Ophthalmol 2010; 21: 293-300.

11 Alexandrakis G, Haimovici R, Miller D, Alfonso EA. Corneal biopsy in the management of pregressive microbial keratitis. Am J Ophthalmol 2000; 129: 571-576.

12 Buchser NM, Dubovy SR, Miller D. Detection of organisms by microbiology vs. histopathology in corneal biopsy and penetrating keratoplasty specimens. Invest Ophthalmol Vis Sci 2009; 50: E-abstract 5119 .

13 Jones DB. Early diagnosis and therapy of bacterial corneal ulcers. Int Ophthalmol Clin 1973 Winter 13(4): 1-29.

14 Kaufman SC, Musch DC, Belin MW, Cohen EJ, Meisler DM, Reinhart WJ et al. Confocal microscopy: a report by the American Academy of Ophthalmology. Ophthalmology 2004; 111(2): 396-406
15 Ford JG, Huang AJ, Pflugfelder SC, Alfonso EC, Forster RK, Miller D. Mycobacterial keratitis in South Florida. Ophthalmology 1998; 105(9): 1652-1658.

16 Srinivasan M, Mascarenhas J, Rajaraman R, Ravindran M, Lalitha P, Glidden DV et al. Corticosteroids for Bacterial Keratitis: the Steroids for Corneal Ulcers Trial (SCUT). Arch Ophthalmol 2011; e-pub ahead of print 10 October 2011; doi:10.1001/archophthalmol.2011.315.

17 Thompson PP, Kowalski RP. A 13-year retrospective review of polymerase chain reaction testing for infectious agents from ocular samples. Ophthalmology 2011; 118(7): 1449-1453.

18 Martins SAR, Combs JC, Noguera G, Camacho W, Wittmann P, Walther R et al. Antimicrobial efficacy of riboflavin/UVA combination (365 n). In vitro for bacterial and fungal isolates: a potential new treatment for infectious keratitis. Invest Ophthalmol Vis Sci 2008; 49: 3402-3408.

19 Iseli HP, Thiel AM, Hafezi F, Kampmeier J, Seiler T. Ultraviolet A/riboflavin corneal cross-linking for infectious keratitis associated with corneal melts. Cornea 2008; 27(5): 590-594.

20 Micelli Ferrari T, Leozappa M, Lorusso M. Escherichia coli keratitis treated with ultraviolet $\mathrm{A} /$ riboflavin corneal cross-linking: a case report. Eur J Ophthalmol 2009; 19(2): 295-297.

21 Marangon FB, Miller D, Alfonso EC. Impact of prior therapy on the recovery and frequency of corneal pathogens. Cornea 2004; 23(2): 158-164.

22 Ventura R, Miller D, Alfonso E. Laboratory update on microbial keratitis in South Florida. ARVO Meeting Abstr 2009; 50: 3115.

23 Yoo SH, Kymionis GD, O'Brien TP, Ide T, Culbertson W, Alfonso EC. Femtosecond-assisted diagnostic corneal biopsy (FAB) in keratitis. Graefes Arch Clin Exp Ophthalmol 2008; 246: 759-762.

24 Deng SX, Kamal KM, Hollander DA. The use of voriconazol in the management of post-penetrating keratoplasty Paecilomyces keratitis. J Ocul Pharmacol Ther 2009; 25(2): 175-177.

25 Utine CA, Stern M, Akpek EK. Clinical Review: topical ophthalmic use of cyclosporine A. Ocul Immunol Inflamm 2010; 18(5): 352-361.

26 Rodman RC, Spisak S, Sugar A. The utility of culturing corneal ulcer in the tertiary referral center verus a general ophthalmology clinic. Ophthalmology 1997; 104(11): 1897-1901.

27 Saini JS, Jain AK, Kumar S, Vikal S, Pankaj S, Singh S. Neural network approach to classify infective keratitis. Curr Eye Res 2003; 27(2): 111-116.

28 Chandna A, Fisher AC, Cunningham I, Stone D, Mitchell M. Pattern recognition of vertical strabismus using an artificial neural network (StrabNet). Strabismus 2009; 17(4): 131-138.

29 Bizios D, Heijl A, Bengtsson B. Trained artificial neural network for glaucoma diagnosis using visual field data: a comparison with conventional algorithms. J Glaucoma 2007; 16(1): 20-28.

30 Kaiserman I, Rosner M, Peer J. Forcasting the prognosis of choroidal melanoma with an artificial neural network. Ophthalmology 2005; 112(9): 1608. 